\title{
Avrami Master Curves for Isothermal Polymer Crystallization
}

\author{
Markus LAMBRIGGER \\ Laboratorium für Festkörperphysik, Swiss Federal Institute of Technology, \\ Im Struppen 12, CH-8048 Zürich, Switzerland
}

(Received August 1, 1996)

\begin{abstract}
KEY WORDS Avrami Master Curves / Inflexion Point / Polymer Crystallization / Kinetics /
\end{abstract}
The kinetic crystallization model of Avrami is the generally accepted starting point for the analysis of isothermal nucleation and crystallization of polymers. ${ }^{1-3}$ The three-parameter Avrami equation function is given by

$$
\theta(t ; n, k, \tau)=\frac{X(t)}{X_{\infty}}=1-\exp \left[-k(t-\tau)^{n}\right]
$$

where $\theta(t)$ is the relative crystallinity at time $t, X(t)$ is the absolute crystallinity at time $t, X_{\infty}$ is the ultimate absolute crystallinity, $k$ is the crystallization rate constant containing the nucleation and growth rates, $\tau$ is the incubation time, and $n$ is the Avrami index. For repeated series of isothermal crystallization experiments run at different temperatures, only the Avrami index $n$ is found to be temperature-independent, ${ }^{4}$ whereas the same is not valid for the two other parameters $k$ and $\tau$. In a previous communication, ${ }^{5}$ the author has already shown that data of isothermal crystallization performed at different temperatures can be evaluated together with the help of Avrami master curves. Moreover, it has been shown that the three-parameter Avrami equation function shows an inflexion point for $n>1.5$ The time value of the inflexion point $t_{\text {in }}$ can be calculated by solving the equation

$$
\frac{\partial^{2} \theta\left(t_{\mathrm{in}}\right)}{\partial t^{2}}=0
$$

Thus, it follows for $n>1$

$$
t_{\text {in }}=\left[\frac{(n-1)}{n k}\right]^{1 / n}+\tau
$$

Introducing a dimensionless time $a$,

$$
a=\frac{(t-\tau)}{\left(t_{\mathrm{in}}-\tau\right)}
$$

a scaled, modified Avrami function $I(a ; n)$ can be obtained:

$$
\theta(t ; n, k, \tau)=I(a ; n)=1-\exp \left\{\left[\frac{(1-n)}{n}\right] a^{n}\right\}
$$

Avrami master curves $I(a ; n)$ are displayed in Figure 1 for $n>1$. Avrami master curves even for $0<n \leq 1 \quad n \approx 1$ is expected for rod-like growth geometries), can be obtained by scaling $\theta(t)$ with the mean-time $\bar{t}$. The meantime $\bar{t}$ is given by

$$
\bar{t}=\int_{0}^{1} t \mathrm{~d} \theta=\tau+\frac{\Gamma\left(1+\frac{1}{n}\right)}{k^{1 / n}}
$$

Formally, eq 6 has already been found by Trustrum and Jayatilaka. ${ }^{6}$ The complete Gamma-function $\Gamma(1+(1 / n))$ is defined by

$$
\Gamma\left(1+\frac{1}{n}\right)=\int_{0}^{\infty} x^{1 / n} \exp (-x) d x
$$

The values of $\Gamma(1+(1 / n))$ are real and positive for every $n>0$. Introducing a new dimensionless time $y$,

$$
y=\frac{t-\tau}{\bar{t}-\tau}
$$

a second type of Avrami master curves $K(y ; n)$ can be obtained:

$$
\theta(t ; n, k, \tau)=K(y ; n)=1-\exp \left\{-\left[\Gamma\left(1+\frac{1}{n}\right)\right]^{n} y^{n}\right\}
$$

Avrami master curves $K(y ; n)$ can be constructed for every real, positive Avrami index $n$. Avrami master curves $K(y ; n)$ for $n>0$ are displayed in Figure 2.

Since the only parameter of the Avrami master curves $I(a ; n)$ and $K(y ; n)$ is the Avrami index $n$, a resp. $y$ being variables, there exists a one-to-one correspondence between the two types of Avrami master curves for every

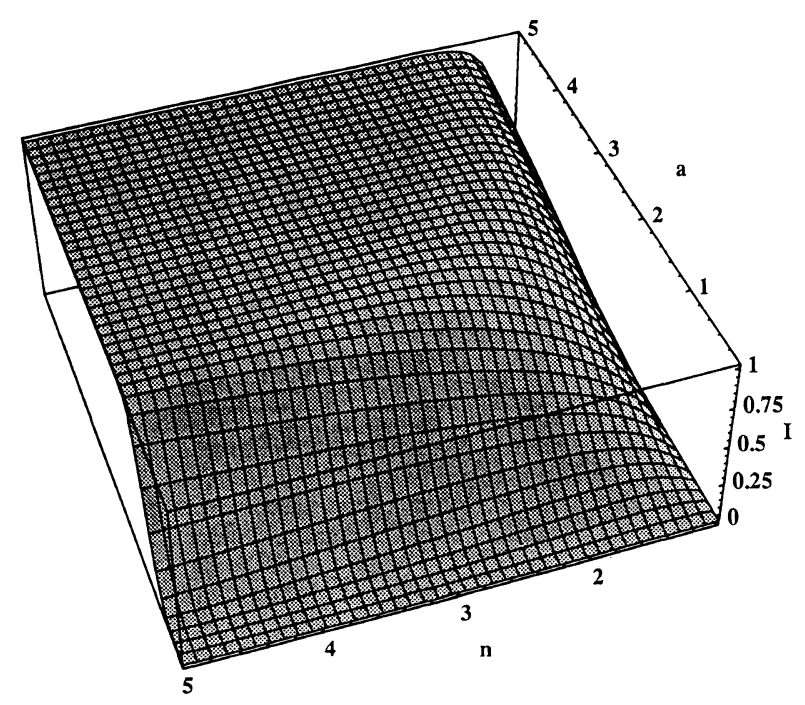

Figure 1. Avrami master curves $I(a ; n)$. 


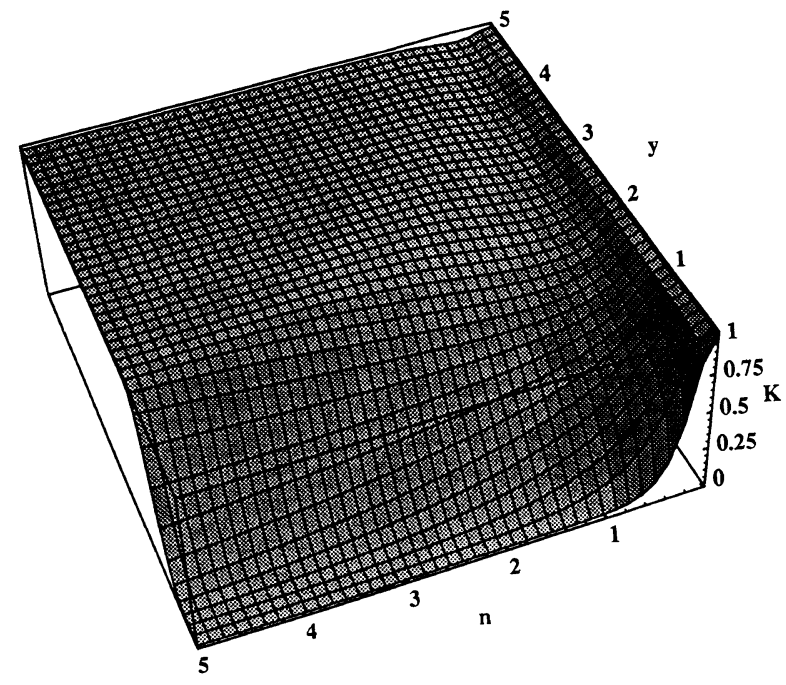

Figure 2. Avrami master curves $K(y ; n)$.

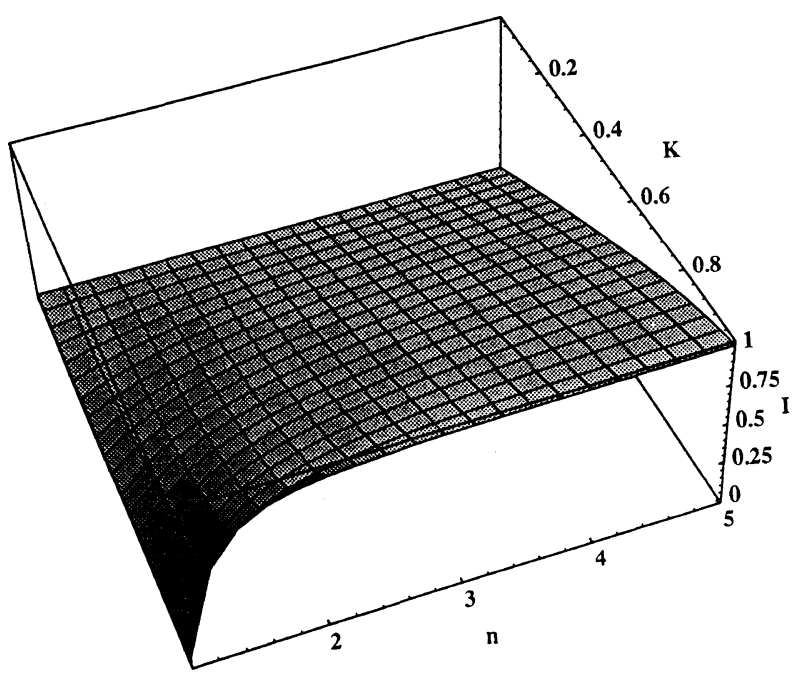

Figure 3. Avrami master curves $I(a ; n)$ as a function of the Avrami index $n$ and the Avrami master curves $K(y ; n)$.

fixed Avrami index $n>1$. The variables $a$ and $y$ can be expressed as follows:

$$
\begin{aligned}
& y=\frac{\{-\ln [1-K(y ; n)]\}^{1 / n}}{\left(\Gamma\left(1+\frac{1}{n}\right)\right)} \\
& a=\left\{\frac{n \ln [1-I(a ; n)]}{1-n}\right\}^{1 / n}
\end{aligned}
$$

Two corresponding master curves $I(a ; n)$ and $K(y ; n)$ can thus be compared to one another at every possible, fixed value of the variables a resp. $y$. This is done, formally, by setting $a=y=$ constant. Thus it follows

$$
\begin{array}{r}
I(c ; n)=1-\exp \left\{\frac{(n-1) \ln [1-K(c ; n)]}{n\left(\Gamma\left(1+\frac{1}{n}\right)\right)^{n}}\right\} \\
K(c ; n)=1-\exp \left\{\frac{n\left(\Gamma\left(1+\frac{1}{n}\right)\right)^{n} \ln [1-I(c ; n)]}{(n-1)}\right\}
\end{array}
$$

Equation 12 is displayed in Figure 3. Equations 12 and 13 express the possibility of computing Avrami master curves $I(a ; n)$, if a master curve $K(y ; n)$ is evaluated from experimental data and vice versa. This is possible as long as the experimentally evaluated Avrami index $n>1$. Furthermore, the Avrami master curves $K(y ; n)$ are physically more significant than the $I(a ; n)$ master curves, since the scaling parameter $\bar{t}$ (mean-time) represents the first moment of the three-parameter Avrami equation function $\theta(t)$. Therefore, the master curves $K(y ; n)$ are as well physically more relevant as more general than the master curves $I(a ; n)$.

In a previous communication, ${ }^{5}$ the author has already displayed a difference quotient method, which enables the evaluation of the parameters $n, k$, and $\tau$, if at least three experimental data points $\left(t_{\mathrm{i}}, \theta\left(t_{\mathrm{i}}\right)\right)$ are available. Therefore, $t_{\text {in }}$ and $\bar{t}$ can be experimentally obtained by evaluating the parameters $n, k$, and $\tau$ and by using eq 3 resp. eq 6 afterwards.

Experimental evidence of the isothermal polymer crystallization theory, based on Avrami master curves, has already been given by the author, ${ }^{5}$ having evaluated experimental data of isothermal crystallization experiments of polydimethylsiloxane.

\section{REFERENCES}

1. M. Avrami, J. Chem. Phys., 7, 1103 (1939).

2. M. Avrami, J. Chem. Phys., 8, 212 (1940).

3. M. Avrami, J. Chem. Phys., 9, 177 (1941).

4. T. W. Chan and A. I. Isayev, Polym. Eng. Sci., 34, 461 (1994).

5. M. Lambrigger, Polym. Eng. Sci., 36, 98 (1996).

6. K. Trustrum and A. De S. Jayatilaka, J. Mater. Sci., 14, 1080 (1979). 